\title{
OpenMemDB: A Wait-Free, In-Memory Database
}

\author{
Michael McGee
}

Robert Medina

\author{
Neil Moore
}

\author{
Jason Stavrinaky
}

\begin{abstract}
OpenMemDB is an in-memory database that is implemented solely using wait-free data structures. OpenMemDB is the first and only database currently developed in such a way. OpenMemDB also provides linearizable correctness guarantees for all operations executed on the database. OpenMemDB uses a form of snapshot isolation to ensure linearizability, and avoids the write-skew problem that can occur when using snapshot isolation by eliminating writes that are out of data. OpenMemDB's biggest contribution is its completely wait-free implementation. Every operation executed in OpenMemDB is guaranteed to be wait-free and linearizable. This implementation also scales competitively when compared against similar in-memory database management systems. OpenMemDB achieves its best scaling in select heavy operation loads with nearly 12 times speedup at 16 threads. This is better scaling than either VoltDB or MemSQL showed in our testing.
\end{abstract}

\section{Introduction}

While hard drives are getting faster due to the introduction of NAND flash-based drives, they are still relatively slow when compared to main memory. Meanwhile main memory is following a long running pattern where it has precipitously dropped in price from costing thousands of dollars for a few megabytes to about $\$ 40$ for 8 gigabytes [6]. With this trend, we can take advantage and design fast databases that reside entirely in main memory. While the largest of datasets are still too large for main memory, some datasets are finally able to fit into main memory in modern systems due to the cheapness and advancement of memory module technology.

Most technologies have advanced along with hardware, however database management systems have struggled to improve at a similar rate. This is mostly due to concurrency issues. Databases spend more than $30 \%$ of execution time in synchronization-related operations, even when only running a single client thread [8]. And in this era, where a powerful server can have more than a terabyte of RAM and well over 64 cores, effectively utilizing all of this processing power is essential.

Our approach, OpenMemDB, solves this problem by implementing a data-store using only wait-free data structures that scale extremely well with added cores. OpenMemDB is a SQL database designed from the ground up to provide fast access to shared data without using any locks. This is achieved through the use of wait-free data-structures provided by Tervel, a library of wait-free and lock-free datastructures developed by Feldman et. al [2] [5] [3]. The lack of contention on locks and the wait-free guarantees that the Tervel data-structures provide will achieve the performance gains that have been lacking in the DBMS field.

OpenMemDB's largest contribution is being the first waitfree database management system. Wait-freedom is a progress guarantee for concurrent data-structures which states that all threads running operations on the data-structure are guaranteed to complete in a finite number of steps. This sort of guarantee can be vital in real-time systems where a hard limit needs to be placed on how long an operation is allowed to take. One situation in which OpenMemDB would be ideal is for use in real-time database systems, where data has a temporal validity, and all calculations using this data must complete within this valid range of time. An example of a real-time database system is a stock market analysis database. A database that is used to track the current state of the stock market and run calculations based on the temporarily valid data.

OpenMemDB is also well suited for any situation in which a large amount of calculations need to be executed on a relatively centralized data-set. This is due to the fact that OpenMemDB provides all threads access to every part of the database. Some database systems attempt to use data partitioning to help parallelize their operations, these systems would not be a good choice if the data-set could not be efficiently partitioned. OpenMemDB does not do any partitioning and still manages to achieve $90 \%$ scaling at 8 threads and $60 \%$ scaling at 32 threads.

OpenMemDB also retains all necessary ACID properties, which is vital for most database systems. The requirements of being wait-free dictate that OpenMemDB is an inmemory database and thus avoids the bottlenecks associated with going to hard disk. OpenMemDB is written in $\mathrm{C}+11$ and uses the modern constructs defined by that standard extensively.

\section{Related Work}

There are several other in-memory database management systems that attempt to solve the problem of data-contention. This is the main source of lowered performance for inmemory systems. 
The database management system most related to OpenMemDB is MemSQL. MemSQL uses lock-free data structures for every component of their database management system. MemSQL uses lock-free skips lists and hash tables for the bulk of their data-store [7]. They also use MVCC (Multi-version Concurrency Control) to provide fast transactions. OpenMemDB does something similar with snapshot isolation. MemSQL also compiles SQL statement to C++ code and stores this code to be used if the SQL statement is ever called again. Something that OpenMemDB does not implement.

VoltDB is an in-memory database management system that uses pure SQL, is completely ACID compliant, and does not use locks. They claim to achieve 100 times the performance of a traditional relation database management system, with near linear scaling with added nodes [10]. They claim to achieve 560,000 transaction per second on a 12 partition set up. VoltDB uses a shared nothing architecture. Their execution engine is single threaded, avoiding the overhead of locking or latching [10]. This requires intelligent partitioning of data and does not allow for multi-threaded execution on a single partition.

Silo is an in-memory database that uses optimistic concurrency control (OCC) to try and limit the effects of locks on their system. Silo avoids locking during the computation of a transaction and waits until commit time to execute all writes to shared memory. This avoids the contention involved in acquiring a lock until a short period of time at the end of transaction. This style of system can be particularly effective if concurrent writes to shared memory are uncommon. Silo claims to achieve 700,000 transaction per second on a 32 core machine. Silo relies heavily on concurrent B+ tree for its back end data-store [9].

Hekaton is Microsoft's main memory optimized database engine. Hekaton uses a combination of lock-free datastructures and optimistic concurrency control in order to achieve scalable performance gains. Hekaton is like Silo and OpenMemDB in that it does not use partitioning to achieve performance gains. Any thread can access any row in a table without acquiring a lock [1]. Hekaton claims to gain an order of magnitude performance gain over standards SQL Server [1]

\section{Technical Approach}

Our database is built upon wait-free data structures found in Tervel, a collection of lock-free and wait-free data structures created by Feldman et al [2] [5] [3]. We use the common definition of wait-freedom as found in Herlihy's definitive text, which states that all threads must make progress within a finite amount of time [4]. OpenMemDB is built to be waitfree and linearizable by utilizing Tervel data structures to create the underlying data structures used by the database to service queries and commands.
OpenMemDB tables are made up of a composition of Tervel data structures, namely the hash map and the vector. The hash map handles the relation of a table's name to its table object. The vectors used in a way that a vector, referred to as the table vector, holds references to other Tervel vectors that contain the actual data used. As only references to records are stored in the table vector, most operations on the table take place as whole record operations rather than a more fine-grained approach that would update records on a percolumn basis. This easily facilitates snapshot isolation and mitigation of the write skew that comes with it.

The datastore uses snapshot isolation to increase read performance and minimize the amount of time accessing the shared data structures. Snapshot isolation is the technique where a thread or transaction copies the data locally and then manipulates it, which avoids performing those manipulations directly on the data structures. A side effect of snapshot isolation is write skew, which occurs when a write operation takes place even though the predicate that determined that record should be written to is no longer valid. We avoid write skew caused by snapshot isolation by only committing the writes if the expected data is in the table. This is done by using the Compare and Swap (CAS) operation provided by Tervel's vector data structure.

The CAS operation checks if the element in the vector is the expected value and swaps the value with the given value if that check succeeds. Tervel's CAS operation is wait-free and atomic, so any contention will have a clear "winner" or "loser" in terms of if a thread succeeds in updating or writing to the vector. This translates to a thread failing to update the table with the new record reference if another thread replaces the record before the attempted write but after the initial read of the record. The loser thread or threads then report that they encountered contention during execution, but continue to perform their operation as best as possible.

\section{Experimental Results}

Our experiments took place on a 64-core AMD Opteron system with $300 \mathrm{~GB}$ of system memory running Ubuntu 14.04 and GCC 4.8.4. Each database was given the same series of operations to perform for each test, with each database connected to locally so as to remove network latency from the test. We explicitly only tested other databases with SQL frontends, as our goal was a SQL database. We tested various workloads in order to evaluate the databases' ability to interleave writes and reads at the same time as well as particularly write-heavy loads. Each test was run with 1, $2,4,8,16,32$, and 64 cores available to the database which maximizes the amount of hardware processors available to the OS but does not exceed it.

We ran three tests on all of the databases: an insert only test, a select only test, and a mixed test that consisted of 
$33 \%$ writes and $66 \%$ reads. The resultant scaling found by running these tests can be seen in the tables below.

TABLE 1: OpenMemDB Scaling

\begin{tabular}{llll}
\hline Threads & Insert & Select & Mixed \\
\hline 2 & 1.84 & 1.93 & 1.94 \\
\hline 4 & 2.94 & 3.08 & 3.54 \\
\hline 8 & 4.19 & 6.97 & 7.33 \\
\hline 16 & 4.98 & 11.87 & 13.10 \\
\hline 32 & 4.75 & 19.05 & 20.44 \\
\hline 64 & 3.16 & 26.03 & 27.25 \\
\hline
\end{tabular}

TABLE 2: MemSQL Scaling

\begin{tabular}{llll}
\hline Threads & Insert & Select & Mixed \\
\hline 2 & 1.20 & 1.90 & 1.68 \\
\hline 4 & 2.31 & 3.49 & 3.31 \\
\hline 8 & 4.53 & 6.26 & 5.63 \\
\hline 16 & 8.54 & 10.77 & 10.80 \\
\hline 32 & 15.13 & 16.84 & 18.21 \\
\hline 64 & 20.18 & 9.43 & 10.30 \\
\hline
\end{tabular}

TABLE 3: VoltDB Scaling

\begin{tabular}{llll}
\hline Threads & Insert & Select & Mixed \\
\hline 2 & 1.75 & 1.61 & 1.42 \\
\hline 4 & 3.58 & 2.70 & 2.83 \\
\hline 8 & 6.13 & 4.51 & 3.93 \\
\hline 16 & 9.12 & 7.97 & 5.98 \\
\hline 32 & 10.71 & 11.92 & 7.12 \\
\hline 64 & 10.56 & 15.30 & 7.27 \\
\hline
\end{tabular}

Table 1 shows the scaling achieved by OpenMemDB for each of the three benchmarks that were run. It is clear OpenMemDB scales much better in read-heavy tests compared to the other databases. This is likely due to a reduction in memory allocations in this test compared to the Insert test, which allocates memory frequently in OpenMemDB. MemSQL has the best scaling of all of the databases in the Insert test that were benchmarked. Table 2 shows the scaling for MemSQL for each of the three benchmarks. MemSQL does not scale as well as OpenMemDB in read-heavy tests, this could be due to a number of factors and further testing is needed to assess the cause. An interesting anomaly is the precipitous drop in scaling after 32 cores, which was repeated in multiple runs of the benchmark. Table 3 shows the scaling for VoltDB for all three benchmarks. It performed consistently in each benchmark though with less scaling than expected. This is likely due to our configuration for these tests are not optimal for VoltDB's architecture.

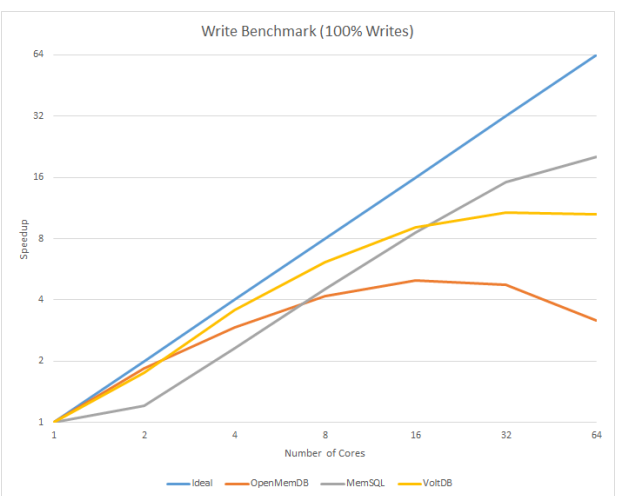

Figure 1: 500,000 writes on the selected databases

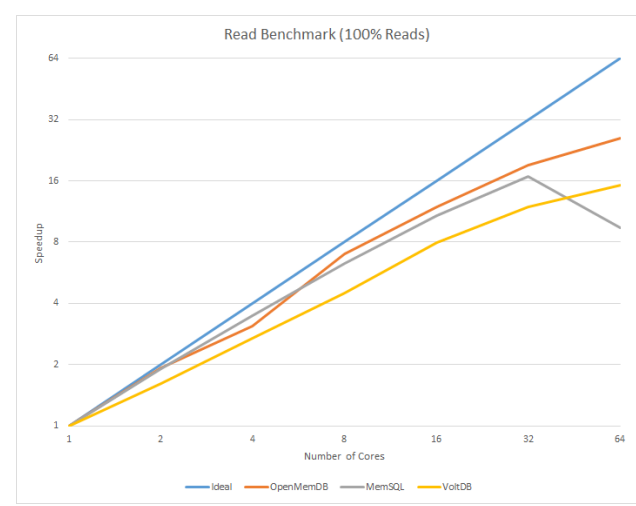

Figure 2: 10,000 reads on the selected databases 


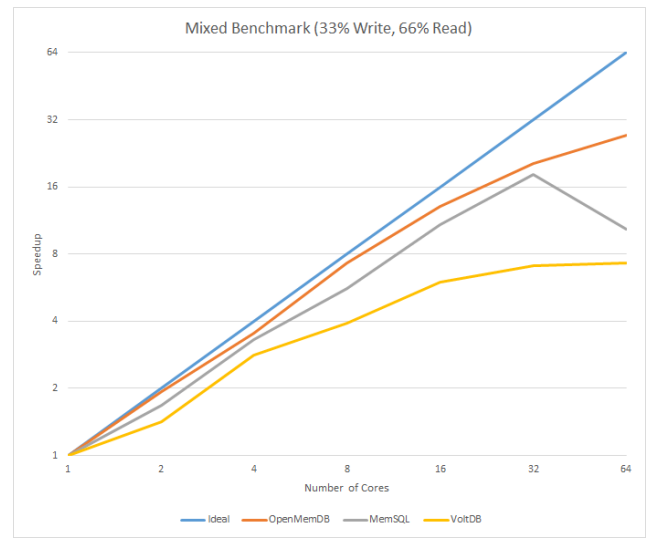

Figure 3: 10,000 mixed reads and writes on the selected databases

\section{Conclusions}

We have presented OpenMemDB, an in-memory and waitfree database that achieves competitive scaling when compared to other in-memory SQL database management system. OpenMemDB scales particularly well with read heavy workloads, achieving 90\% scaling at 8 threads and $60 \%$ scaling at 32 threads when running our read operation tests. OpenMemDB is the first, and only fully wait-free database in existence at the time of writing this paper.

Based on the encouraging scaling achieved, further work to improve individual thread performance would bring this database closer to commercial viability, in particular with embedded systems where consistent latency is preferable to faster average performance with higher amounts of jitter in latency.

Some of the areas that can be improved with more time would be: improving the memory allocation model to reduce thread synchronization, further optimizations of the tokenization and parsing of SQL statements, and improved support for standard SQL operations and data types.

\section{References}

[1] Diaconu, C., Freedman, C., Ismert, E., Larson, P.-A., Mittal, P., Stonecipher, R., Verma, N., ANd Zwilling, M. Hekaton: Sql server's memory-optimized oltp engine. In Proceedings of the 2013 ACM SIGMOD International Conference on Management of Data (2013), ACM, pp. 1243-1254.

[2] Feldman, S., LaBorde, P., And Dechev, D. Tervel: A unification of descriptor-based techniques for non-blocking programming. In Embedded Computer Systems: Architectures, Modeling, and Simulation (SAMOS), 2015 International Conference on (2015), IEEE, pp. 131-140.

[3] Feldman, S., Valera-Leon, C., and Dechev, D. An efficient wait-free vector. IEEE Transactions on Parallel and Distributed Systems 27, 3 (March 2016), 654-667.

[4] Herlihy, M., And Shavit, N. The Art of Multiprocessor Programming, 1st ed. Morgan Kaufmann, June 2012.

[5] Laborde, P., Feldman, S., And Dechev, D. A wait-free hash map. International Journal of Parallel Programming (2015), 1-28.

[6] McCallum, J. C. Memory prices 1957 to 2015, 2015.

[7] MeMSQL. MemSQL Docs Home, 2015.

[8] SoARes, J., ANd PreguiçA, N. Database engines on multicores scale: A practical approach.

[9] Tu, S., Zheng, W., Kohler, E., Liskov, B., And Madden, S. Speedy transactions in multicore in-memory databases. In Proceedings of the Twenty-Fourth ACM Symposium on Operating Systems Principles (2013), ACM, pp. 18-32.

[10] VoltDB, L. Voltdb technical overview. Whitepaper (2010). 